\title{
The therapeutic effect of gelatin tannate in acute diarrhea in children
}

\author{
Soner Sertan Kara ${ }^{1}$, Burcu Volkan ${ }^{2}$, İbrahim Erten ${ }^{3}$ \\ Divisions of ${ }^{1}$ Pediatric Infectious Diseases and ${ }^{2}$ Pediatric Gastroenterology, ${ }^{3}$ Department of Pediatrics, Regional Training \\ and Research Hospital, Erzurum, Turkey. E-mail: drsoner@yahoo.com \\ Received: 4th March 2017, Revised: 7th June 2017, Accepted: 4th July 2017
}

SUMMARY: Kara SS, Volkan B, Erten İ. The therapeutic effect of gelatin tannate in acute diarrhea in children. Turk J Pediatr 2017; 59: 531-536.

Gelatin tannate (GT) is a nonabsorbable antidiarrheal agent investigated in few clinical studies. The aim of this study was to investigate the effects of GT on children with acute gastroenteritis. This randomized, placebocontrolled, single-blinded, prospective study involved children aged from six months to 10 years with acute diarrhea. The study group received GT and the control group placebo for five days. Stool frequency and numbers of patients with diarrhea in each group were compared at $12,24,48,72,96$, and 120 hours. Duration of diarrhea and weight changes after 120 hours was recorded. Mean stool frequency was lower in the study group at 0-12 hours $(3 \pm 1.8$ vs. $3.6 \pm 1.9, p=0.04)$. The study group exhibited more weight gain after 120 hours of treatment and shorter total duration of diarrhea, although the difference was not statistically significant. Fewer patients in the study group had diarrhea at the end of 12, 24, 96, and 120 hours. Patients treated with GT with Bristol scores of 7 at admission exhibited more weight gain than patients with Bristol scores of $6(296 \pm 38$ vs. $137 \pm 39, p=0.04)$. GT resulted in a decreased stool frequency at 12 hours in children with acute diarrhea. It shortened total duration of diarrhea and resulted in more weight gain compared to placebo. It also had a greater effect on weight gain in the presence of watery, rather than mushy stool.

Key words: acute diarrhea, children, gelatin tannate, rotavirus.

Acute gastroenteritis (AGE) is a common cause of morbidity and mortality worldwide. One billion new cases and nearly 3 million AGErelated deaths occur in children under 5 years of age across the world each year. ${ }^{1}$ Rotavirus is the most common cause of infectious diarrhea in children worldwide, followed by adenovirus and norovirus. ${ }^{2}$ There is not an effective treatment for viral gastroenteritis, except for suitable vaccines and implementation of infection control measures. Although cases of viral AGE are usually easy to prevent and self-limiting, dehydration and eventual hospitalization constitute a significant burden, especially on pediatric populations. Additionally, high prevalence and consequences such as poor growth, impaired cognitive development, and environmental enteropathy further emphasize its significance in terms of public health. ${ }^{3}$

The management of children and infants with viral AGE mainly depends on oral or intravenous hydration, breastfeeding, and early refeeding. Probiotics are also beneficial in acute infectious diarrhea by shortening duration and reducing stool frequency. ${ }^{4}$ There is convincing evidence of the beneficial nature of some of these in particular. ${ }^{5,6}$ Gelatin tannate (GT) is an antidiarrheal medical device that is not absorbed in the gastrointestinal system. It is composed of protective gelatin and tannic acid and possesses astringent, antibacterial, and anti-inflammatory properties. It precipitates proinflammatory proteins and prevents local

This manuscript was presented as a poster presentation in the 52 nd Turkish Pediatrics Congress, Kemer/Antalya, May 15-19, 2016. This manuscript was presented as an e-poster presentation in the $50^{\text {th }}$ Annual Congress of ESPGHAN, Prague/Czech Republic, May 10-13, 2017. 
inflammation. ${ }^{7}$ GT is a promising alternative for use alone or in combination with probiotics because of its relatively safer profile, fewer drug interactions, and rapid effect on stool frequency in children. ${ }^{8}$ However, because of lack of randomized clinical studies, it is not strongly recommended in guidelines. ${ }^{5}$

The aim of this study was to investigate the effects of GT on clinical characteristics of children with viral AGE, such as the frequency of diarrhea episodes on each day, total duration of diarrhea, and changes in body weight.

\section{Materials and Methods}

This randomized, placebo-controlled, singleblinded, prospective study was performed with children aged from six months to 10 years in our hospital. Children with acute diarrhea for the previous 12 to 72 hours were included. Diarrhea was defined as the passage of three or more loose or watery stools per day. ${ }^{9}$ Exclusion criteria included severe dehydration, concomitant use of any antidiarrheal (probiotics or any drugs affecting gastrointestinal motility or absorption), antibiotic or immunosuppressive agents, and presence of any underlying conditions (severe malnutrition, immunodeficiency, and chronic diseases).

All patients were first examined by a pediatrician. Assessment included anthropometric measurements, physical examination, and Modified Vesikari and CDS scores. Eligible patients with viral AGE were randomized on the basis of order of presentation, the first to

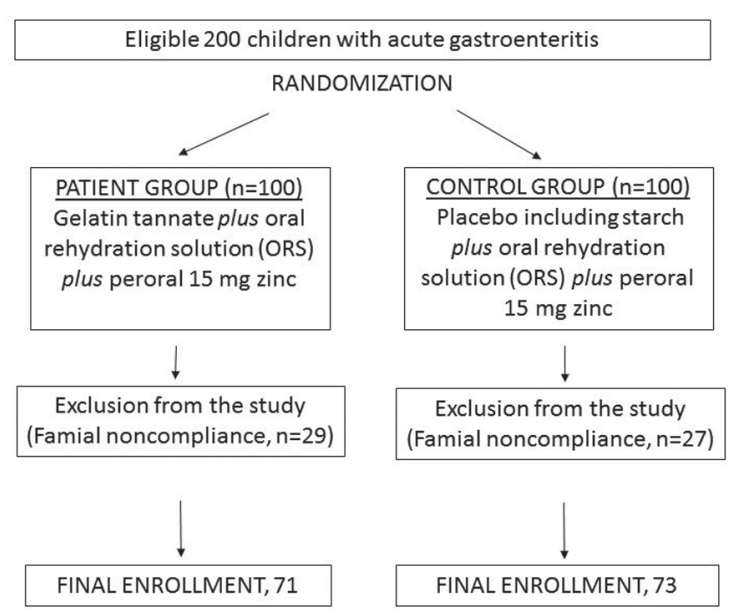

Fig. 1. Study population flow chart. present being included in the patient group, and the second in the control group, etc. The care-givers were blinded to the treatment type. The study group received GT (Tasectan ${ }^{\circledR}$, Onko-Koçsel, Turkey, $250 \mathrm{mg}$ sachet) four times a day plus oral rehydration solution (ORS) (glucose 20 g; sodium $90 \mathrm{mEq} / \mathrm{L}$; potassium 20 $\mathrm{mEq} / \mathrm{L}$; bicarbonate $30 \mathrm{mmol} / \mathrm{L}$ ) plus peroral zinc (15 mg/day) for five days. The control group received $250 \mathrm{mg}$ maltodextrin containing placebo sachets four times a day plus ORS plus peroral zinc for five days. The parents were given a Bristol stool chart, and stool frequency and consistency were checked by their mobile phone on a daily basis. Number of and consistency of stools were recorded at 12, 24, $48,72,96$, and 120 hours. Duration of diarrhea was determined according to Bristol criteria, with a score lower than 5 being described as normal stool. ${ }^{10}$ Control examination was carried out at the end of 120 hours.

Stool samples at admission were evaluated for Salmonella, Shigella, Campylobacter, Clostridium difficile, adenovirus, rotavirus, norovirus, Entamoeba histolytica, and Giardia lamblia. Stool cultures detected bacterial pathogens while microscopic examination was performed in order to determine parasitic infestations. Clostridium difficile toxin A and B were tested by a commercial qualitative enzyme immunoassay method (The Serazym ${ }^{\circledR}$ Clostridium difficile Toxin A+B ELISA, Pfungstadt, Germany) in stool specimens. Rotavirus and adenovirus antigens were analyzed using a combined kit Ecotest ${ }^{\circledR}$ Rotavirus and Adenovirus Combo Rapid test device (D-RAC-52) (Assure Tech, Zhejiang, China), according to the manufacturer's instructions. Stool samples were

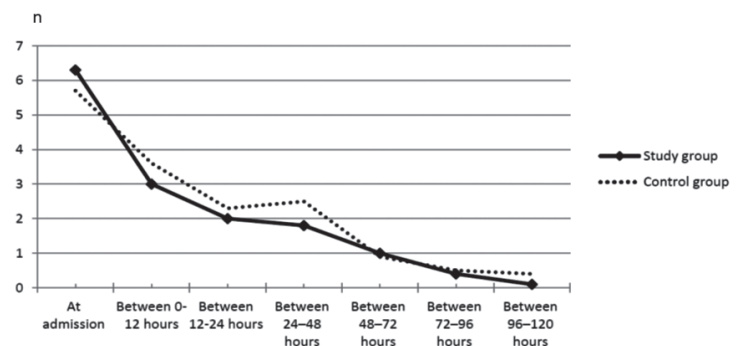

Fig. 2. Mean number of stools per day at different time points according to the groups. Gelatin tannate group had significantly lower number of stools per day, compared to controls at 12 hours $(3 \pm 1.8$ vs. $3.6 \pm 1.9, p=0.04)$. The groups were comparable for other time points. 
examined for Norovirus using the Rida ${ }^{\circledR}$ Quick Norovirus test (R-Biopharm AG, Darmstadt, Germany) in line with the manufacturer's instructions. Children not complying with the study protocol were excluded (Fig. 1).

The primary endpoints were numbers of stools at 12, 24, and 48 hours. The total duration of diarrhea (in hours), changes in body weight at the $120^{\text {th }}$ hour of intervention, and the number of patients with diarrhea at each defined interval were assessed as the secondary endpoints.

The study protocol was approved by local ethical committee and Turkish Medicines and Medical Devices Agency. Written informed consent was obtained from parents of all children.

\section{Statistical analyses}

The Kolmogorov-Smirnov and Shapiro-Wilk tests were used to determine the normality of the data. Descriptive statistical analysis of the data was performed using measures of central tendency (mean and median) and dispersion (standard deviation and range). Univariate analysis was performed to compare differences among the study groups using the chi-square, independent samples t-test, and Mann-Whitney $\mathrm{U}$ tests. $\mathrm{P}$ values of $<0.05$ were considered statistically significant. All statistical analyses were performed on SPSS 17.0 software (SPSS
Inc.; Chicago, IL).

\section{Results}

Seventy-one children were included in the study group, while 73 children were included in the control group. No differences were determined between the children in the study and control groups in terms of age, anthropometric measurements, admission Vesikari and CDS scores, numbers of diarrhea and vomiting episodes during the previous 24 hours, or duration of diarrhea until admission. A significant difference was detected between groups only in terms of gender [male gender, $\mathrm{n}=42 \quad(59.1 \% 9$ vs. $\mathrm{n}=31 \quad(42.4 \%) ; \mathrm{p}=0.04)$ (Table I).

The children in the study and control groups were compared in terms of mean stool frequencies at time intervals $0-12$ hours, 12 - 24 hours, $24-48$ hours, $48-72$ hours, 72 - 96 hours, and 96 - 120 hours. Mean number of stools was lower in the study group than in the control group at $0-12$ hours $(3 \pm 1.8$ vs. $3.6 \pm 1.9, \mathrm{p}=0.04$ ) (Fig. 2). Although the differences were not statistically significant, the study group exhibited more weight gain at the end of 120 hours of treatment and a shorter total duration of diarrhea (Table II). Additionally, while the difference was again not

Table I. Clinical Characteristics of the Study Population on Admission.

\begin{tabular}{lccc}
\hline Parameter & $\begin{array}{c}\text { Study group } \\
(\mathrm{n}=71)\end{array}$ & $\begin{array}{c}\text { Control group } \\
(\mathrm{n}=73)\end{array}$ & $\mathrm{p}$ \\
\hline Age (months) & $44.2 \pm 35.7$ & $44.1 \pm 31.8$ & 0.79 \\
Z-score of weight & $-0.77(-6.40-2.33)$ & $-0.59(-4.04-[-0.59])$ & 0.10 \\
Z-score of height & $0.15(-7.97-2.52)$ & $0.19(-4.69-3.89)$ & 0.33 \\
Z-score of BMI & $-1.21(-3.88-2.29)$ & $-0.70(-5.47-1.51)$ & 0.06 \\
CDS score & $0(0-4)$ & $0(0-4)$ & 0.35 \\
Modified Vesikari score & $8.2 \pm 2.9$ & $8.3 \pm 2.6$ & 0.60 \\
Duration of diarrhea until admission (hours) & $38.6 \pm 23.3$ & $35.4 \pm 19.1$ & 0.51 \\
Number of diarrhea* & $6.3 \pm 3.3$ & $5.7 \pm 3.2$ & 0.19 \\
Number of vomiting & $2(0-20)$ & $3(0-20)$ & 0.22 \\
Diarrhea etiology, n (\%) & & & \\
$\quad$ Rotavirus & $10(6.9 \%)$ & $20(13.8 \%)$ & \\
$\quad$ Adenovirus & $2(1.3 \%)$ & $4(2.7 \%)$ & \\
$\quad$ Norovirus & $4(2.7 \%)$ & $0(0 \%)$ & \\
$\quad$ Unidentified\# & $55(38.1 \%)$ & $49(34.0 \%)$ & \\
\hline
\end{tabular}

Data is presented as mean \pm standard deviation or median (min-max), according to the distribution of variable. *: number of episodes during the previous 24 hours; \#: stool samples negative for Salmonella, Shigella, Campylobacter, Clostridium difficile, adenovirus, rotavirus, norovirus, Entamoeba histolytica, and Giardia lamblia BMI: body mass index, CDS: clinical dehydration scale 
Table II. Duration of Diarrhea and its Impact on Body Weight During the Study Period.

\begin{tabular}{llll}
\hline Parameter & $\begin{array}{l}\text { Study group } \\
(\mathrm{n}=71)\end{array}$ & $\begin{array}{l}\text { Control group } \\
(\mathrm{n}=73)\end{array}$ & $\mathrm{p}$ \\
\hline Total duration of diarrhea (hours) & $42(6-260)$ & $44(8-360)$ & 0.38 \\
Weight gain at 120 hours (grams) & $200(-500-1380)$ & $100(-1000-1100)$ & 0.12 \\
\hline
\end{tabular}

Data is presented as median (min-max)

significant, fewer patients had diarrhea at the end of $12,24,96$, and 120 hours in the study group than in the control group (Table III).

The study patients with a stool consistency compatible with a Bristol score of 7 at admission exhibited greater weight gain at 120 hours than those with a stool consistency compatible with a Bristol score of 6 at admission (296 \pm $38 \mathrm{~g}$ vs. $137 \pm 39 \mathrm{~g} ; \mathrm{p}=0.04)$. However, no significant difference was found between the control patients in terms of stool consistency or weight gain at 120 hours.

An etiological agent was determined in stool samples from 40 patients (Table I). Rotavirus was the agent in the majority of these. Nevertheless, no further statistical investigation was able to be performed due to the scarcity of case numbers.

No adverse events were recorded in any patients.

\section{Discussion}

In this study, children with viral AGE who were treated with GT had fewer diarrhea episodes at the end of the first 12 hours of treatment than children treated with placebo. No differences were determined between the groups in terms of age, anthropometric measurements, admission dehydration scores, numbers of diarrhea and vomiting episodes or duration of diarrhea until admission. Esteban Carretero et al. ${ }^{8}$ reported similar results in children. However, they described a significant difference between the numbers of stools at admission. That study also showed that the stool decrease index [(number of frequency of stools at 12 hours - number of frequency of stools at admission)/frequency of stools at admission] was significantly lower in the group of children treated with GT plus ORS. Allegrini et al. ${ }^{11}$ also examined the effects of GT in adult AGE patients and reported that the GT group produced a similar daily number of watery stools on day 1 , but a reduced number on day 2, compared with the placebo group. Probiotics, a well-established choice for diarrhea treatment, have been reported to reduce the total duration of diarrhea in approximately one day and to lower stool frequency on day $2 .{ }^{4}$ Meta-analyses regarding the use of $S$. boulardii in the treatment of acute infectious diarrhea revealed that it shortened the initial phase of watery stools and started to reduce the mean number of stools on day 2, a longer time than exhibited by GT.12,13 Similarly there is strong evidence that Lactobacillus GG reduces the duration of diarrhea in children with AGE. ${ }^{14}$ These probiotics both receive a strong degree of recommendation in the ESPGHAN Working Group for Probiotics and Prebiotics report. ${ }^{15}$ Other probiotics, such as Lactobacillus reuteri DSM 17938, have been reported to exhibit promising effects in reducing the incidence and duration of acute diarrhea in AGE in children. ${ }^{16,17}$ Nevertheless, and similarly to GT, no strong recommendation for their use has yet been published.

Tannic acid and gelatin are the components of GT, which is not absorbed, and which is excreted from intestines without causing any systemic effects. In vitro studies have shown its protective effects on membranous barriers against chemical erosion. ${ }^{18}$ It acts as a mechanical barrier in the intestinal walls, thereby stabilizing intestinal permeability, mucus layer integrity, and colonic microbiota variety. ${ }^{19}$ This barrier blocks increased tight junction permeability in AGE with no effects on enterocytes. In the present study, the patients in the study group with a Bristol score of 7 at admission exhibited greater weight gain at the end of the 5th day of intervention than the study patients with a Bristol score of 6 . This finding can also be interpreted as GT being particularly successful in preventing loss of water or possibly some catch-up weight gain in the study patients.

Although the difference was not statistically 
Table III. Percentages of Children with Diarrhea During the Study Period According to Groups.

\begin{tabular}{lcccc}
\hline Time & $\begin{array}{c}\text { Study Group* } \\
(\mathrm{n}=71)\end{array}$ & $\begin{array}{c}\text { Control Group* } \\
(\mathrm{n}=73)\end{array}$ & RR (CI 95\%) & $\mathrm{p}$ \\
\hline At 12 hours & $59(83.0 \%)$ & $67(91.7 \%)$ & $0.91(0.80-1.03)$ & 0.11 \\
At 24 hours & $45(63.3 \%)$ & $55(75.3 \%)$ & $0.84(0.67-1.05)$ & 0.11 \\
At 48 hours & $31(43.6 \%)$ & $25(34.2 \%)$ & $1.27(0.84-1.93)$ & 0.24 \\
At 72 hours & $13(14.0 \%)$ & $13(17.8 \%)$ & $1.03(0.51-2.56)$ & 0.93 \\
At 96 hours & $7(9.8 \%)$ & $10(13.6 \%)$ & $0.72(0.29-1.79)$ & 0.47 \\
At 120 hours & $2(2.8 \%)$ & $7(9.5 \%)$ & $0.27(0.05-1.36)$ & 0.47 \\
\hline
\end{tabular}

*: data is presented as $\mathrm{n}(\%), \mathrm{CI}$ : confidence interval, RR: relative risk

significant, the mean total duration of diarrhea was shorter and weight gain was greater in the GT group in this study. Unfortunately, no comparable results are available from previous GT studies regarding these parameters. ${ }^{8,11} \mathrm{~S}$. boulardii has been reported to reduce the number of stools and risk of diarrhea at days 2, 3, and 4. ${ }^{12,13}$ In the present study, fewer patients in the study group had diarrhea at the end of 12 , 24, 96, and 120 hours of the treatment than control patients, which may also suggest the efficacy of GT.

Rotavirus was the most frequently detected etiological agent in this study, and is also the most common cause of diarrhea in children worldwide. Approximately $95 \%$ of children experience rotavirus gastroenteritis by the age of 5 years, and the agent exhibits no predilection for developed or developing countries. ${ }^{20}$ The positive effects of probiotics have previously been shown to reduce the duration of acute rotavirus diarrhea compared with control patients. ${ }^{14,21}$ Nevertheless, a comparison could not be performed between the groups in this study in terms of rotavirus etiology.

There are a number of limitations to this study. First, it was not a double-blinded clinical trial, and the design of the study made it necessary for parents to perform the evaluation of stool frequency and consistency. This may not have resulted in an optimal assessment and may have yielded lower accuracy. The outcome measurements were a comparison of the groups in terms of stool frequencies and weight gain during the study period, as in most clinical trials in AGE. It would have been preferable to have included other parameters, such as stool volume, frequency of vomiting, and changes in appetite. Additionally, AGE cases due to non-viral pathogens were excluded in order to avoid treatment bias. Although, only a limited number of viral agents were determined in this study, the rest of all included diarrhea episodes were compatible with viral AGE with respect to stool properties and symptoms of the patients. It was known that the antigen detection EIA or ELISA of the enteropathogenic viruses has some limitations in sensitivity and specificity rather than molecular testing, but the patients were compared with regard to rotavirus and pathogen-specific effects of GT for other viruses could not be evaluated.

This study provides evidence that GT exhibits a rapid ability, in as little as 12 hours, to reduce stool frequency. It shortens the total duration of diarrhea and results in greater weight gain compared to placebo in children with AGE. Additionally, it exhibited a clear therapeutic effect in AGE in terms of weight gain in the presence of watery stool, rather than mushy stool. The results of the study should now be confirmed with larger scale clinical studies. Further studies addressing pathogen-specific effects of GT, cost-effectiveness of intervention with GT, and therapeutic effects of GT when combined with prebiotics should also be designed.

\section{REFERENCES}

1. Baker T. Burden of community diarrhoea in developing countries. Lancet Glob Health 2016; 4: e25.

2. Wiegering V, Kaiser J, Tappe D, Weissbrich B, Morbach H, Girschick HJ. Gastroenteritis in childhood: A retrospective study of 650 hospitalized pediatric patients. Int J Infect Dis 2011; 15: e401-e407.

3. Platts-Mills JA, Babji S, Bodhidatta L, et al. Pathogenspecific burdens of community diarrhoea in developing countries: A multisite birth cohort study (MAL-ED). Lancet Glob Health 2015; 3: e564-e575. 
4. Allen SJ, Martinez EG, Gregorio GV, Dans LF. Probiotics for treating acute infectious diarrhoea. Cochrane Database Syst Rev 2010; CD003048.

5. Guarino A, Ashkenazi S, Gendrel D, et al. European Society for Pediatric Gastroenterology, Hepatology, and Nutrition; European Society for Pediatric Infectious Diseases. European Society for Pediatric Gastroenterology, Hepatology, and Nutrition/European Society for Pediatric Infectious Diseases evidencebased guidelines for the management of acute gastroenteritis in children in Europe: Update 2014. J Pediatr Gastroenterol Nutr 2014; 59: 132-152.

6. Cruchet S, Furnes R, Maruy A, et al. The use of probiotics in pediatric gastroenterology: A review of the literature and recommendations by Latin-American experts. Paediatr Drugs 2015; 17: 199-216.

7. Ruszczyński M, Urbańska M, Szajewska H. Gelatin tannate for treating acute gastroenteritis: A systematic review. Ann Gastroenterol 2014; 27: 121-124.

8. Esteban Carretero J, Durbán Reguera F, López-Argüeta Alvarez S, López Montes J. A comparative analysis of response to ORS (oral rehydration solution) vs. ORS + gelatin tannate in two cohorts of pediatric patients with acute diarrhea. Rev Esp Enferm Dig 2009; 101: 41-48.

9. King CK, Glass R, Bresee JS, Duggan C; Centers for Disease Control and Prevention. Managing acute gastroenteritis among children: Oral rehydration, maintenance, and nutritional therapy. MMWR Recomm Rep 2003; 52: 1-16.

10. Lewis SJ, Heaton KW. Stool form scale as a useful guide to intestinal transit time. Scand J Gastroenterol 1997; 32: 920-924.

11. Allegrini A, Costantini M. Gelatine tannate for the treatment of acute diarrhoea in adults. J Gastroint Dig Syst 2012; 2: 3.

12. Dinleyici EC, Eren M, Ozen M, Yargic ZA, Vandenplas Y. Effectiveness and safety of Saccharomyces boulardii for acute infectious diarrhea. Expert Opin Biol Ther 2012; 12: 395-410.
13. Feizizadeh S, Salehi-Abargouei A, Akbari V. Efficacy and safety of Saccharomyces boulardii for acute diarrhea. Pediatrics 2014; 134: e176-e191.

14. Szajewska H, Skórka A, Ruszczyński M, GieruszczakBiałek D. Meta-analysis: Lactobacillus GG for treating acute gastroenteritis in children-updated analysis of randomised controlled trials. Aliment Pharmacol Ther 2013; 38: 467-476.

15. Szajewska H, Guarino A, Hojsak I, et al. Use of probiotics for management of acute gastroenteritis: A position paper by the ESPGHAN Working Group for probiotics and prebiotics. J Pediatr Gastroenterol Nutr 2014; 58: 531-539.

16. Dinleyici EC, PROBAGE Study Group, Vandenplas Y. Lactobacillus reuteri DSM 17938 effectively reduces the duration of acute diarrhoea in hospitalised children. Acta Paediatr 2014; 103: e300-e305.

17. Dinleyici EC, Dalgic N, Guven S, et al. Lactobacillus reuteri DSM 17938 shortens acute infectious diarrhea in a pediatric outpatient setting. J Pediatr (Rio J) 2015; 91: 392-396.

18. Bueno L. Mechanisms involved in bacterial pathogeninduced intestinal mucosal barrier disruption: Therapeutic interest of physical protection of epithelial tight junction. WebEx Conference, June 25, 2014.

19. Scaldaferri F, Lopetuso LR, Petito V, et al. Gelatin tannate ameliorates acute colitis in mice by reinforcing mucus layer and modulating gut microbiota composition: Emerging role for 'gut barrier protectors' in IBD? United European Gastroenterol J 2014; 2: 113-122.

20. Bernstein DI. Rotavirus overview. Pediatr Infect Dis J 2009; 28: S50-S53.

21. Ahmadi E, Alizadeh-Navaei R, Rezai MS. Efficacy of probiotic use in acute rotavirus diarrhea in children: A systematic review and meta-analysis. Caspian J Intern Med 2015; 6: 187-195. 